\title{
MEASURES ON LOCALLY COMPACT TOPOLOGICAL GROUPS
}

\author{
BY \\ W. AMBROSE
}

Introduction. In this paper we show that every left invariant measure on a locally compact topological group $G$, which is a "refinement" of the Haar measure $m$ of $G$ and satisfies André Weil's measurability conditions( $\left.{ }^{1}\right)$, gives rise to a locally compact subgroup of $G$. Imposing certain nontriviality conditions on the refinement of $m$ we can further ensure that the locally compact subgroup we obtain is nontrivial, in the sense that it is not discrete and has $m$-measure 0 . We also determine, in a sense, the most general refinement of $m$ (satisfying Weil's measurability conditions).

Our original concern with this question came from the following observation. If $G$ is a locally compact group and $H$ a locally compact subgroup then $H$ has a Haar measure, and "transplanting" that measure on $H$ to all the left cosets of $H$ in $G$ gives a measure on $G$, which in general is a "refinement" of the Haar measure of $G$. Then we asked whether this procedure could be reversed, that is, given a measure on $G$ which is a refinement of the Haar measure of $G$, can it be used to get a locally compact subgroup. If so, this would be a possible method of getting locally compact subgroups of $G$. This paper contains the answer to the reverse problem for measures satisfying Weil's conditions, by showing that such a "refinement" of the Haar measure always gives rise to a locally compact subgroup (however the relation between the measure and the subgroup is more complicated than that mentioned above, for the given measure need not always be of the kind obtained by transplanting the Haar measure of a locally compact subgroup). The subgroup we obtain need not be unique, but what is unique is a locally compact topology on $G$ which is a refinement of the given topology, and in which $G$ is again a topological group. Then we get a subgroup by taking any compact neighborhood of the identity and the subgroup generated by it.

It is desirable to prove a corresponding theorem under more general hypotheses because the measure on $G$ obtained by transplanting the Haar measure of a locally compact subgroup does not always satisfy the Weil conditions, as Oxtoby has shown $\left({ }^{2}\right)$, so we can not hope to obtain all locally compact subgroups by our process. We believe that it will be possible to reduce the general

Presented to the Society, September 15, 1945; received by the editors February 25, 1946.

(1) $[4$, pp. 140-146]. Numbers in brackets refer to the bibliography.

(2) [5]. We had the good fortune to read Oxtoby's paper in unpublished form while completing this paper. Prior to reading Oxtoby's paper we had not noticed this fact. Oxtoby discusses conditions under which Weil's conditions will be satisfied. 
case to the case considered here and intend to take that up at a later date.

A particularly simple example of the situation we are concerned with is the following: Let $G$ be the Euclidean plane, whose Haar measure is ordinary two-dimensional Lebesgue measure. Consider the "refinement" of $m$ which assigns to every linearly measurable set on the real axis, or any parallel line, its linear measure. This measure (considered as a measure on the plane) is associated in a natural way with the locally compact subgroup, the real axis, and with that topology on the plane in which the neighborhoods of any point are the horizontal linear intervals about the point.

Our result depends on and is closely related to the fundamental theorem of A. Weil that an invariant measure on a group $G$ (satisfying certain measurability conditions) gives rise in a natural way to a locally conditionally compact topology, so that the group can then be completed to a locally compact group. Our work is even more closely related to a paper of Kodaira [2] dealing with this Weil theorem and related results. In particular, our procedure for getting a locally compact topology from the given refinement was introduced by Kodaira. Also, Kodaira has considered refinements of a measure, though with such restrictive countability assumptions as to eliminate the matters that are our main concern $\left({ }^{3}\right)$. Our definition of a refinement is not the same as his.

This paper is divided into three sections. $\$ 1$ contains definitions and statements of known facts, plus one theorem asserting that our notion of a refinement of a measure is essentially the same thing, for locally compact groups, as the corresponding notion of a refinement of the topology. $\$ 2$ is concerned solely with getting a locally compact topology from a locally conditionally compact topology (not by completing the space, but by changing the topology) and $\S 3$ uses $\$ 2$ to derive our main results. At one point in $\S 3$ (the proof of Theorem 3.4) we need a certain direct sum theorem for Haar measures. This is proved in the following paper.

1. Preliminaries. Most of this section is devoted to definitions and though many of the concepts are well known we give the definitions in full. We do this because on certain points not all authors agree, and these points sometimes make an essential difference. For example, omitting from the domain of definition of a measure the fact that it is closed under complements and including property (4) of Definition 2 below makes the Fubini theorem hold much more generally than it is usually stated, as Weil has pointed out [4, pp. 31-33].

Definition 1. Let $X$ be a space (that is, any set of points). A $\sigma$-field $\Sigma$

(8) Kodaira always assumes that the whole space is a countable union of measurable sets of finite measure. This assumption occurs in his definitions on p. 70, and for groups follows from his Axiom (B), p. 75. Hence the "horizontal linear measure" on the plane described in the preceding paragraph is not a measure for him. Since this is typical of the situation we are interested in, our results have little in common. 
is a collection of subsets of $X$ closed under the operations of difference and countable union, that is, if $E \in \Sigma$ and $F \in \Sigma$ then $E-F \in \Sigma$ and if $E_{n} \in \Sigma$ $(n=1,2, \cdots)$ then $U_{n} E_{n} \in \Sigma$.

Definition 2. Let $X$ be any space. A measure on $X$ is a function $m E$ with the following properties:

(1) The domain of $m$ is some $\sigma$-field $\Sigma$ of subsets of $X$.

(2) The range of $m$ is included in the set of non-negative real numbers together with the symbol $\infty$, and for some $E \in \Sigma$ we have $m E \neq 0$.

(3) $m$ is countably additive, that is, if $E=\cup_{n} E_{n}$ where $E_{n} \in \Sigma(n=1$, $2, \cdots)$ and $E_{n} \| E_{m}\left({ }^{4}\right)$ for $n \neq m$ then $m E=\Sigma_{n} m E_{n}$.

(4) Every set in $\Sigma$ is a countable union of sets of finite measure, that is, of sets $E_{n} \in \Sigma$ with $m E_{n}<\infty$.

(5) If $E \in \Sigma$ and $m E=0$ then every subset of $E$ is again in $\Sigma$.

The space $X$ together with the measure $m$ is called a measure space, the sets in $\Sigma$ are called measurable sets, and $m E$ is called the measure of $E$.

Condition 5 in this definition is not essential but it simplifies uniqueness statements. A measure with this property is of ten called a completed measure. If $E$ is any subset of $X$ we define the inner measure of $E$ to be the sup $m F$ where this sup is taken over all measurable $F$ contained in $E$.

Definition 3. Let $X$ be a measure space and $X_{1}$ a subset of $X . X_{1}$ is thick in $X \mathrm{ff}$ the complement of $X_{1}$ in $X$ has inner measure 0 .

Definition 4. Let $X$ be a measure space with a measure $m$ and let $X_{1}$ be a thick subset of $X$. We define a measure $m_{1}$ on $X_{1}$, called the measure induced by $m$, as follows:

(1) The domain of $m_{1}$ is the $\sigma$-field of all sets of the form $E_{1}=X_{1} \cap E$, where $E$ is $m$-measurable.

(2) For any such set we define $m_{1} E_{1}$ to be $m E\left(^{(5)}\right.$.

This is the last of our purely measure-theoretic definitions and we now turn to invariant measures on groups.

Definition 5. Let $G$ be a group. A left-invariant measure on $G$ is a measure $m$ on $G$ with the property: for every measurable set $E$ and every $x \in G$ the set $x E$ is also measurable and $m(x E)=m E$.

A. Weil has shown that with every measurable (see definition below) left invariant measure is associated a unique measurable right invariant measure $[4$, p. 148] so it doesn't matter whether we work with right or left invariant measures. All our measures will be left invariant. We state now, for future reference, the standard theorem about the existence and uniqueness of Haar measure.

THEOREM. If $G$ is a locally compact topological group then there exists on $G$

(4) $E \| F$ means that the sets $E$ and $F$ have an empty intersection.

(s) It is of course necessary to know that if $E_{1}$ is represented like this in two ways, those two ways assign the same measure to $E_{1}$. This was proved by Doob [1] in a special case and is easily seen to be true in general. 
a unique (to within a constant factor) left invariant measure for which all compact sets are measurable and of finite measure, and whose $\sigma$-field of definition is the $\sigma$-field generated by the compact sets (after subsets of sets of measure 0 have been included). This measure is called the Haar measure of $G$, and its measurable sets are called Haar-measurable. Every open Haar-measurable set has positive measure.

A. Weil has proved a sort of converse to this theorem asserting that if a group $G$ has a left invariant measure satisfying certain conditions then $G$ is "almost" locally compact, the "almost" meaning that $G$ is a thick subgroup of a locally compact group $G^{*}$, with the given measure on $G$ being that induced by the Haar measure on $G^{*}[4, \mathrm{pp} .140-146]$. We shall use this theorem, so we state it below. The next definition gives the name "measurable measure" to a measure satisfying Weil's conditions.

Definition $6\left(^{6}\right)$. A left invariant measure on a group $G$ is measurable $\mathrm{ff}$ it satisfies the two conditions:

(1) The transformation of $G \times G$ onto itself defined by $(x, y) \rightarrow(x, x y)$ is measure preserving (with respect to the independent product measure on $G \times G)$.

(2) For every $x \in G, x \neq e$, there is a measurable set $M$ of finite positive measure such that $m(M \cap x M) \neq m(M)$.

The second of these conditions has nothing to do with measurability, really; it is just a topological separation axiom in measure-theoretic terms $\left({ }^{7}\right)$. We include it because we do not wish to consider other measures.

Weil has shown that the Haar measure of a locally compact topological group is always measurable [4, p. 41]. An example of à nonmeasurable measure (which, however satisfies condition (2) above) is Carathéodorey linear measure on the plane. This example, and the proof I cite here that it is nonmeasurable, was told to me by J. C. Oxtoby. It is trivial that the Weil topology (see definition below) associated with this measure is the discrete topology on the plane, so that if this were a measurable measure it would have to be the Haar measure of the plane in the discrete topology $\left({ }^{8}\right)$ - which it is not.

Definition 7. Let $m$ be a measurable left invariant measure on the group $G$. By the Weil topology associated with $m\left({ }^{9}\right)$ we mean the topology on $G$ obtained by taking the sets of the form $M M^{-1}$, with $M$ measurable and of finite positive measure, for a determining collection of neighborhoods of the identity.

We can now state the Weil theorem mentioned above.

(8) These conditions are taken from [4, pp. 141 and 143]. We use "ff" to mean "if and only if "-a notation due to P. R. Halmos.

(7) This is pointed out in [4, pp. 142-143].

(8) This is a special case of $[4$, p. 146].

(9) Weil shows that these sets do give a topology on $G$, and one nicely related to the given measure $[4$, p. 142]. This is of course well known for locally compact groups. 
THEOREM [4, pp. 141-146]. If $G$ is a group and $m$ a measurable left invariant measure on $G$ then $G$ is a thick subgroup of a locally compact topological group $G^{*}$, whose Haar measure induces m-measure on $G$, and whose topology induces on $G$ the Weil topology associated with $\mathrm{m}$.

We shall want to use the term "topological subgroup" in more general situations than that where the topology of the subgroup is the one induced by the topology of the whole group. Hence we make the following definition.

Definition 8. Let $G$ be a topological group and $H$ a subgroup of $G$ which is also a topological group in some topology of its own. $H$ is a topological subgroup of $G$ ff the identity mapping of $H$ into $G$ is continuous.

An equivalent way of stating this is to say that the given topology on $H$ is a refinement of the topology on $H$ induced from the topology on $G$. By a locally compact subgroup we shall mean a topological subgroup which is locally compact in its own topology.

Definition 9. Let $G$ be a group with left invariant measures $m$ and $n$. Then $n$ is a refinement of $m$ (denoted by $n \geqq m$ ) ff every $m$-measurable set of positive $m$-measure contains an $n$-measurable set of positive $n$-measure.

For example, let $G$ be the plane and $m$ its ordinary two-dimensional Lebesgue measure, which is the Haar measure of this group. Let $n$ be defined as follows: the $n$-measurable sets are those of the form $\bigcup_{n} E_{n}$ (disjoint countable union) where each $E_{n}$ is a linearly Lebesgue measurable subset of some horizontal line in the plane. The $n$-measure of such a set.is the sum of the linear measures of the $E_{n}$ sets. We call $n$ the "horizontal" linear measure in the plane, and $n$ is "induced" on the plane in an obvious way by the Lebesgue (Haar) measure of the subgroup, the real line. Clearly $n$ is a refinement of $m$, and with any line through the origin in any direction we have similarly an induced measure on the plane which is a refinement of $m$. Clearly any locally compact topological subgroup $H$ of a locally compact topological group $G$ gives rise in this same fashion to a refinement of the Haar measure of $G$.

The following theorem justifies our definition of a refinement of the Haar measure.

THEOREM 1.1(10). Let $G$ be a group which is a locally compact topological group in a topology $T$ and also in a topology $U$. Let the Haar measure of $G$ in these topologies be denoted by $m$ and $n$, respectively. Then $U$ is a refinement of $T$ $f f$ is a refinement of $m$.

Proof. First we prove that $n \geqq m$ implies $U \geqq T\left({ }^{11}\right)$. It is sufficient to prove that every $T$-neighborhood of $e$ contains a $U$-neighborhood of $e$, so let $O$ be any $T$-neighborhood of $e$. Then choose $O_{1}$ a $T$-neighborhood of $e$ such that

(10) Compare [2, pp. 111-113].

(1i) We use $U \geqq T$ to mean that the $U$-topology is a refinement of the $T$-topology, that is, that every $T$-open set is $U$-open. 
$O_{1} O_{1}^{-1} \leqq 0$. Being $T$-open $O_{1}$ must have positive $m$-measure; hence by the assumption that $n \geqq m$ we can choose an $n$-measurable set $N$ of positive $n$-measure such that $N \subseteq O_{1}$. By a well known theorem $N N^{-1}$ contains a $U$-neighborhood of $e$, and since $N N^{-1} \subseteq O_{1} O_{1}^{-1} \subseteq O$ this $U$-neighborhood is contained in the given $O$. Hence $U$ is a refinement of $T$.

To show that $U \geqq T$ implies $n \geqq m$ we shall actually prove the following lemma, which is clearly stronger.

Lemma 1.1. Assume the hypotheses of Theorem 1.1. If $M$ is any m-measurable set of finite positive m-measure and $N$ is any $n$-measurable set of finite positive n-measure, then $z N \cap M$ is n-measurable for all $z$, except possibly a set of $m$-measure 0 , and for some $m$-measurable set of $z$ 's of positive $m$-measure the set $z N \cap M$ is $n$-measurable and of positive $n$-measure.

Proof. We consider the transformation $\psi$ :

$$
\psi:(x, y) \rightarrow(x, x y)
$$

of $G \times G$ onto itself. We consider $G \times G$ in the $U \times T$-topology and use $n \times m$ measure on $G \times G\left({ }^{12}\right)$. Because $U$ is a refinement of $T, \psi$ is continuous, and hence takes compact sets into compact sets. Also the Fubini theorem shows that $\psi$ preserves the measure of compact sets. Since the compact sets generate the measurable sets it then follows by standard arguments that $\psi$ is a measure preserving transformation, that is, that it takes measurable sets into measurable sets, preserving their measure.

Now let $N$ and $M$ be the given sets. Then $M^{-1}$ is also $m$-measurable and of positive $m$-measure. We consider the set $M^{*}=\psi\left(N \times M^{-1}\right)$, and let $M_{y}^{*}=\left[x \mid(x, y) \in M^{*}\right]$. Because $\psi$ is measure preserving the Fubini theorem implies that $M_{y}^{*}$ is $n$-measurable except possibly for a set $E$ of $y$ 's of $m$-measure 0 , and for a set $P$ of $y$ 's of positive $m$-measure is $n$-measurable and of positive $n$-measure. We have

$$
\begin{aligned}
M_{\nu}^{*} & =\left[x \mid(x, y) \in M^{*}\right] \\
& =\left[x \mid \psi^{-1}(x, y) \in N \times M^{-1}\right] \\
& =\left[x \mid\left(x, x^{-1} y\right) \in N \times M^{-1}\right] \\
& =\left[x \mid x \in N \text { and } x^{-1} y \in M^{-1}\right] \\
& =[x \mid x \in N \text { and } x \in y M] \\
& =N \cap y M .
\end{aligned}
$$

Because $n$ is left invariant it follows that, for $z \notin E^{-1}, z N \cap M$ is $n$-measurable,

(12) For the theory of product measures see [3, pp. 82-88]. The countability hypotheses there are of course too strong, but when everything is modified in accordance with our definitions then it all goes through in our case. In all these modifications to avoid countability restrictions we are of course merely following [4]. 
and for $z \in P^{-1}$ it has positive $n$-measure. Since $E^{-1}$ must have $m$-measure $O$ and $P^{-1}$ must have positive $m$-measure the lemma is proved.

We could have proved the last theorem and lemma under more general conditions. The proof that $n \geqq m$ implies $U \geqq T$ holds if we only assume that $n$ and $m$ are left invariant measurable measures on $G$ and that $U$ and $T$ are their associated Weil topologies. The proof of the converse, that $U \geqq T$ implies $n \geqq m$, holds in this more general situation if we add the assumption that $\psi$ is measure preserving in the sense of $n \times m$-measure on $G \times G$. This condition, incidentally, seems the natural one to use in the definition of a "measurable subgroup" of a "measurable group," where a "measurable group" would be a group with a measurable left invariant measure. That is, if $G$ were such a measurable group with measure $m, H$ a subgroup of $G$ which was a measurable group in its own right, and $n$ the measure on $G$ induced (in the sense of the definition below) by the measure on $H$, then $H$ would be called a "measurable subgroup" of $G \mathrm{ff} \psi$ preserved $n \times m$-measure. We shall not make use of this concept however.

Definition 10. Let $G$ be a group and $H$ a subgroup which has a left invariant measurable left invariant measure $n_{1}$. The measure on $G$ induced by $n_{1}$ on $G$ is the smallest extension of $n_{1}$ to a left invariant measure on $G$.

By the smallest extension we mean the measure with the smallest domain of definition. The existence of such a measure is obtained by simply "transplanting" the given $n_{1}$ to all the left cosets of $H$ in $G$. More precisely: Consider the $\sigma$-field of all sets of the form $N=\mathrm{U}_{n} x_{n} N_{n}$, where the $x_{n}$ belong to distinct left cosets of $H$, the $N_{n}$ are $n_{1}$-measurable subsets of $H$, and the union is countable. The induced measure $n$ is the measure defined on this $\sigma$-field whose value at the set $N$ is defined to be $\sum_{n} n_{1}\left(N_{n}\right)$. To justify this definition we need to know that if $x N_{1}=y N_{2}$, where $x$ and $y$ are in the same left coset of $H$, and $N_{1}$ and $N_{2}$ are $n$-measurable, then $n_{1}\left(N_{1}\right)=n_{1}\left(N_{2}\right)$. To see this note that $x=y h, h \in H$, hence $h N_{1}=N_{2}$; then the left invariance of $n_{1}$ on $H$ guarantees that $n_{1}\left(N_{1}\right)=n_{1}\left(N_{2}\right)$. Oxtoby has shown that the measurability of $n_{1}$ does not imply the measurability of $n$ on $G$.

2. The locally compact topology associated with a refinement of $m$. Let $G$ be a locally compact topological group with topology $T$ and Haar measure $m$ and let $n$ be a refinement of $m$. Then $n$-measure gives rise to an associated Weil topology $U$ on $G$ and we are now going to use both $T$ and $U$ to define still another topology, $U_{1}$, on $G$. It will be characterized by the following properties: (1) $G$ is a locally compact topological group in the $U_{1}$-topology, (2) $T \leqq U_{1} \leqq U$, (3) $U_{1}$ is the finest topology having properties (1) and (2). The third property shows that $U_{1}$ is intrinsically associated with $n, U$ and $T$. The procedure we use to obtain this topology was introduced by Kodaira [2, p. 113].

In $\$ 3$ we shall consider the measures $m$ and $n$ with the above situation but for the present the measures are irrelevant. Here we use only topological 
properties, which certainly hold whenever the topologies are related to measures as in the preceding paragraph. We fix the following notation for the remainder of this section:

$G=$ group.

$T=$ locally compact topology in which $G$ is a topological group.

$U=$ locally conditionally compact topology on $G$, such that $U \geqq T$ and $G$ is a topological group in the $U$-topology.

$G^{*}=$ completion of $G$ from the $U$-topology.

$U^{*}=$ the topology on $G^{*}$.

$H^{*}=$ that subset of $G$ consisting of all points $x$ with the property: every $U^{*}$-neighborhood of $x$ intersects every $T$-neighborhood of $e$ in $G$ ( $e$ the identity element of $G$ and $G^{*}$ ).

In this situation we know of course that $G$ is $U^{*}$-dense in $G^{*}$ and that the $U$-topology on $G$ is that induced by the $U^{*}$-topology on $G^{*}$. The aim of this section is to prove the following theorem.

THEOREM 2.1. $H^{*}$ is a closed invariant subgroup of $G^{*}$, and $G^{*} / H^{*}$ is algebraically isomorphic to $G$, under a natural isomorphism $\phi$. If $U_{1}$ is the topology on $G$ induced from $G^{*} / H^{*}$ under $\phi$ then of course $G$ is a locally compact topological group in the $U_{1}$-topology. Also $T \leqq U_{1} \leqq U$ and if $W$ is any locally compact topology on $G$ in which $G$ is a topological group and such that $T \leqq W \leqq U$ then $W \leqq U_{1}$.

We break the proof into a number of lemmas.

Lemma 2.1. $G \cap H^{*}=(e)$.

Proof. If $x \in G$ and $x \neq e$ then there exist $T$-neighborhoods of $e$ and $x, O_{1}$ and $O_{2}$, with $O_{1} \| O_{2}$. Since $O_{2}$ is also a $U$-neighborhood of $x$ and $U$ is the topology induced by $U^{*}$, the lemma follows.

Lemma 2.2. $H^{*}$ is a $T^{*}$-closed subset of $G^{*}$.

Proof. If $x \in H^{*}$ then there exists a $U^{*}$-neighborhood $O^{*}$ of $x$ and a $T$-neighborhood $O$ of $e$ such that $O^{*} \| O$. Then all points of $O^{*}$ are also outside $H^{*}$. Thus the complement of $H^{*}$ is open, so $H^{*}$ is closed.

LEMMA 2.3. $H^{*}$ is a subgroup of $G$.

Proof. Let $x, y$ be in $H^{*}$, and let $O^{*}$ be any $U^{*}$-neighborhood of $x y, O$ any $T$-neighborhood of $e$. Choose $O_{1}^{*}, O_{2}^{*}, U^{*}$-neighborhoods of $x$ and $y$, respectively, such that $O_{1}^{*} O_{2}^{*} \subseteq O^{*}$, and choose $O_{1}, O_{2}$ any $T$-neighborhoods of $e$ such that $O_{1} O_{2} \subseteq O$. Then $O_{1}^{*} \# O_{1}$ and $\left.O_{2}^{*} \# O_{2}{ }^{18}\right)$. Let $u \in O_{1} \cap O_{1}^{*}, v \in O_{2} \cap O_{2}^{*}$. Then $u v \in O_{1}^{*} O_{2}^{*} \cap O_{1} O_{2} \subseteq O^{*} \cap O$. Hence $O^{*} \# O$, so we have $H^{*} H^{*} \subseteq H^{*}$.

Let $x \in H^{*}, O^{*}$ be any $U^{*}$-neighborhood of $x^{-1}$, and $O$ be any $T$-neighbor-

(18) $E \# F$ means that the sets $E$ and $F$ have a non-empty intersection. 
hood of $e$. Then $O^{*-1} \# O^{-1}$, hence $O^{*} \# O$. Hence $H^{*-1} \subseteq H$. Thus $H^{*}$ is a subgroup of $G^{*}$.

Lemma 2.4. $H^{*}$ is an invariant subgroup of $G^{*}$.

Proof. We first prove that $x \in G$ and $y \in H^{*}$ imply $x^{-1} y x \in H^{*}$. Let $O^{*}$ be any $U^{*}$-neighborhood of $x^{-1} y x$ and $O$ any $T$-neighborhood of $e$. Choose a $T$-neighborhood $O_{1}$ of $x$ and a $T$-neighborhood $O_{2}$ of $e$ such that $O_{1}^{-1} O_{2} O_{1} \subseteq O$, and choose a $U^{*}$-neighborhood $O_{1}^{*}$ of $x$ and a $U^{*}$-neighborhood $O_{2}^{*}$ of $y$ such that $O_{1}^{*-1} O_{2}^{*} O_{1}^{*} \subseteq O^{*}$. Then $O_{2}^{*} \# O_{2}$, and $O_{1}^{*} \# O_{1}$ because $O_{1}^{*} \cap G$ and $O_{1}$ are both $U$-neighborhoods of $x$. Take $x_{1} \in O_{1} \cap O_{1}^{*}$ and $y_{1} \in O_{2} \cap O_{2}^{*}$. Then $x_{1}^{-1} y x_{1} \in O_{1}^{-1} O_{2} O_{1} \subseteq O$ and $x_{1}^{-1} y x_{1} \in O_{1}^{*-1} O_{2}^{*} O_{1}^{*} \subseteq O^{*}$, proving that $O^{*} \sharp O$. Hence $x^{-1} y x \in H^{*}$.

Now we use the fact that $G$ is $U^{*}$-dense in $G^{*}$ to conclude that $x^{-1} y x \in H^{*}$ for all $x \in G^{*}$. Let $O^{*}$ be any $U^{*}$-neighborhood of $x^{-1} y x$ and choose a $U^{*}$-neighborhood $O_{1}^{*}$ of $x$ such that $O_{1}^{*-1} y O_{1}^{*} \subseteq O^{*}$. Let $x_{1} \in O_{1}^{*} \cap G$. Then $x_{1}^{-1} y x_{1} \in O^{*}$, and $x_{1}^{-1} y x_{1} \in H^{*}$ by the preceeding paragraph. Hence every $U^{*}$-neighborhood of $x^{-1} y x$ intersects $H^{*}$. Since $H^{*}$ is closed this implies $x^{-1} y x \in H^{*}$. Hence $H^{*}$ is an invariant subgroup of $G$.

Lemma 2.5. $G H^{*}=G^{*}$, that is, every $x \in G^{*}$ is expressible in the form $y h$, where $y \in G$ and $h \in H^{*}$.

Proof. The first part of this proof finds the $y$ that works. Take $x \in G^{*}$ and let $O^{*}$ be a $U^{*}$-neighborhood of $x$ whose $U^{*}$-closure is $U^{*}$-compact. Then consider $C$, the $T$-closure of $O^{*} \cap G$. $C$ will be a $T$-compact subset of $G$ because $O^{*} \cap G$ is $U$-conditionally compact, hence $T$-conditionally compact, so that $C$ is the $T$-closure of a $T$-conditionally compact set. Now we consider all the $U^{*}$-open sets $O_{1}^{*}$ containing $x$ and contained in $O^{*}$ and for each we consider the corresponding $C_{1}$, the $T$-closure of $O_{1}^{*} \cap G$. Then the $C_{1}$ 's are a family of $T$-closed sets included in the $T$-compact set $C$. Because the $C_{1}$ 's clearly have the finite intersection property there must be a $y$ common to them all. We have thus found, for the given $x \in G^{*}$, a $y \in G$ with the property: for every $U^{*}$-open set containing $x, y$ belongs to the closure of $O^{*} \cap G$.

Now we prove that every $U^{*}$-neighborhood of $x$ intersects every $T$-neighborhood of $y$. Let $O^{*}$ be any $U$-neighborhood of $x^{*}$, and $O$ any $T$-neighborhood of $y$. Then $y$ is in the $T$-closure of $O^{*} \cap G$, hence that $T$-closure intersects $O$. Thus $O^{*} \cap G$ itself must intersect $O$.

We now define $h$, for the given $x$, by $h=y^{-1} x$, where the $y$ is the one found above. We shall show that $h \in H^{*}$. Since obviously $y h=x$ this will prove the lemma. Let $O^{*}$ be any $U^{*}$-neighborhood of $h$ and $O$ any $T$-neighborhood of $e$. Then $y O^{*}$ is a $U$-neighborhood of $y h=x$ while $y O$ is a $T$-neighborhood of $y$. By the preceeding paragraph $y O^{*}$ intersects $y O$, hence $O^{*} \# O$.

Lemma 2.6. Every $x \in G^{*}$ is uniquely expressible in the form $x=y h$, where $y \in G, h \in H^{*}$. 
Proof. This is a trivial consequence of Lemmas 1 and 5 .

LEMMA 2.7. The following three statements are equivalent:

(1) $H^{*}=(e)$.

(2) $G^{*}=G$.

(3) $G$ is U-locally compact.

Proof. From the definition of $G^{*}$ we know that (2) and (3) are equivalent. That $G^{*}=G$ implies $H^{*}=(e)$ is a consequence of Lemma 1 . Now suppose that $H^{*}=(e)$; then, by Lemma $5, G^{*}=G$.

We now recall the purely group-theoretic fact that if $H$ is a group with subgroups $H_{1}$ and $H_{2}$, where $H_{2}$ is invariant, $H_{1} H_{2}=H$, and $H_{1} \cap H_{2}=(e)$ then the natural homomorphism $\psi$ of $H$ onto $H / H_{2}$ establishes an isomorphism between $H_{1}$ and $H, H_{2}$. We denote this isomorphism, considered as a mapping from $H / H_{2}$ onto $H_{1}$, by $\phi$, so $\phi$ is the inverse of the contraction of $\psi$. We shall consider this isomorphism with $G^{*}$ for $G, G$ for $H_{1}$, and $H^{*}$ for $H_{2}$. The reason for considering $\phi$ is that it gives us the desired $U_{1}$-topology on $G$. Because $G^{*}$ is locally compact and $H^{*}$ a closed invariant subgroup we have in the standard way a locally compact topology on $G^{*} / H^{*}$ and because $\phi$ is an isomorphism of $G^{*} / H^{*}$ with $G$ it induces a topology on $G$. This will be our $U_{1}$-topology.

Definition. We denote by $\phi$ the isomorphism of $G^{*} / H^{*}$ onto $G$ discussed above which takes each coset $y H^{*}(y \in G)$ into $y$. We call $\phi$ the natural isomorphism of $G^{*} / H^{*}$ onto $G$.

Definition. The $U_{1}$-topology on $G$ is that induced on $G$ by $\phi$ from the usual topology of the quotient group $G^{*} / H^{*}$.

We have now proved the first two statements of Theorem 2.1. The remainder of the proof consists of proofs that various topologies are related in certain ways. The next four lemmas show that $T \leqq U_{1}$.

Lemma 2.8. If $O$ is a $T$-open set in $G$, containing $e$, then $O H^{*}$ contains a $U^{*}$-neighborhood of $e$.

Proof. Let $E$ be the complement of $O$ in $G$ and choose $O_{1}$ a $T$-neighborhood of $e$ such that $O_{1} \| E O_{1}$. We shall show that if $O^{*}$ is any $U^{*}$-neighborhood of $e$ for which $O^{*} \cap G \subseteq O_{1}$ then $O^{*} \subseteq O H$.

Let $x \in O^{*}, x=y h, y \in G, h \in H^{*}$. Suppose $x \in E H^{*}$. Since $y^{-1} O^{*} \ni y$ we have, by the definition of $H^{*}, y^{-1} O^{*} \# O_{1}$; hence $y O \# O^{*}$, so $E O_{1} \# O$. Thus $E O_{1} \# O_{1}$, contrary to the choice of $O_{1}$.

Lemma 2.9. If $O$ is any $T$-open set in $G$ then $O H^{*}$ is $U^{*}$-open.

Proof. Let $x=y h \in O H^{*}\left(y \in O, h \in H^{*}\right)$. Then $y^{-1} O$ is a $T$-open set contain= ing $e$, hence $y^{-1} O H^{*}$ contains a $U^{*}$-neighborhood of $e$, by Lemma 8 . Then $O H^{*}$ contains a $U^{*}$-neighborhood $O^{*}$ of $y$, so $y h \in O^{*} h \subseteq O H^{*} h=O H^{*}$. Thus $O^{*} h$ is a $U^{*}$-neighborhood of $x$ contained in $O H^{*}$. Hence $O H^{*}$ is open. 
LemMA 2.10. The homomorphism of $G^{*}$ onto $G$ which takes each $x=y$ h ( $x \in G^{*}, y \in G, h \in H$ ) into $y$ is continuous from the $U^{*}$-topology to the T-topology.

Proof. This is merely a rewording of Lemma 2.9.

Lemma 2.11. The $U_{1}$-topology on $G$ is a refinement of the T-topology.

Proof. Let $O$ be any $T$-open subset of $G$; we must show that $O$ is also $U_{1}$-open. By Lemma 2.9, $O H^{*}$ is $U^{*}$-open in $G^{*}$, hence its image in $G^{*} / H^{*}$ under the natural homomorphism of $G^{*}$ onto $G^{*} / H^{*}$ is open in the quotient topology. Since this image maps under $\phi$ exactly onto $O$ again we have proved the lemma.

The last statement of Theorem 2.1 remains to be proved. We prove first that $U \geqq U_{1}$, then use this to prove that if $W$ is any locally compact topology such that $U \geqq W \geqq T$ and $G$ is a topological group in the $W$-topology, then $U_{1} \geqq W$. These are accomplished in the following two lemmas and with them Theorem 2.1 is completely proved.

Lemma 2.12. The $U$-topology is a refinement of the $U_{1}$-topology.

Proof. If $O$ is any $U_{1}$-open set in $G$ then $O H^{*}$ is a $U^{*}$-open set in $G^{*}$ (using the fact that the natural homomorphism of $G^{*}$ onto $G^{*} / H^{*}$ is continuous), hence its intersection with $G$ is open in the $U$-topology, but this intersection is exactly $O$. Thus $O$ is $U$-open.

LEMMA 2.13. If $W$ is a locally compact topology on $G$ in which $G$ is a topological group and if $U \geqq W \geqq T$ then $U_{1} \geqq W$.

Proof. Making use of the previous lemmas with $W$ in place of $T$ we find a subgroup $K^{*}$ of $G^{*}$ that is related to $W$ precisely as $H^{*}$ is related to $T$. Then considering $G^{*} / K^{*}$, we know it is algebraically isomorphic to $G$ and induces a topology $W_{1}$ on $G$-precisely as the $U_{1}$-topology was obtained from $G^{*} / H^{*}$. We know that $W_{1} \geqq W$ (because we proved in Lemma 2.11 that $U_{1} \geqq U$ ) so if we prove that $W_{1}=U_{1}$ then, by Lemma 2.11 , we shall have $U_{1} \geqq W$. To prove $W_{1}=U_{1}$ it will be sufficient to prove $H^{*}=K^{*}$, since then $G^{*} / H^{*}$ and $G^{*} / K^{*}$ will be the same.

Now we prove that $H^{*}=K^{*}$. First, because $W \geqq T$, it is immediate that $K^{*} \subseteq H^{*}$. Then because each $x \in G$ is uniquely expressible in the form $x=y h$ $\left(y \in G, h \in H^{*}\right)$ and also in the form $x=z k\left(z \in G, k \in K^{*}\right)$ it is trivial that $H^{*}=K^{*}$.

3. The general form of a refinement of the Haar measure and the procedure for getting a locally compact subgroup from such a refinement. First we mention some examples of refinements. If $G$ is a locally compact topological group with Haar measure $m$ and if $H$ is any locally compact subgroup then $H$ has a Haar measure, and transplanting this measure to all the cosets of $H$ in $G$ we obtain a measure $n$ on $G$ which is a refinement of $m$. More generally, 
if in the above situation $H_{0}$ is a thick subgroup (in the sense of the Haar measure of $H$ ) of $H$ then we have a measure on $H_{0}$ (that induced by the Haar measure of $H$ ) and transplanting this measure to all cosets of $H_{0}$ in $G$ we obtain a refinement $n_{0}$ of $m$; in general we shall of course have $n \neq n_{0}$. One might suspect that this second type of refinement is the most general type, but it is not. Kodaira has shown [2, pp. 92-93] that there exists a thick subgroup of the plane which contains exactly one point on each vertical line, and the projection $(x, y) \rightarrow x$ gives an isomorphism of this subgroup onto the line. Using the measure induced on this thick subgroup by Lebesgue measure in the plane one obtains a measure on the line which is a refinement of its usual Haar measure, but which is not of the above types. The most general kind of refinement (and it is easy, using Kodaira's procedure, to see that such refinements exist) is described in the following theorem.

THEOREM 3.1. Let $G$ be a locally compact topological group with Haar measure $m$ and lopology $T$. Let $n$ be any measurable left invariant measure on $G$ which is a refinement of $m$. Then there exists a locally compact topological group $G^{*}$ of which $G$ is a subgroup, and a closed invariant subgroup $H^{*}$ of $G^{*}$ such that $G$ contains exactly one point in each coset of $H^{*}$, for which the following are true:

(1) The natural mapping $\phi$ of $G$ onto $G^{*} / H^{*}$ (taking each coset of $G^{*} / H^{*}$ into the point of $G$ lying in that coset) is an isomorphism of $G^{*} / H^{*}$ onto $G$.

(2) $G$ is a thick subgroup of $G^{*}$ in the sense of the Haar measure $m^{*}$ of $G^{*}$.

(3) $n$-measure on $G$ is the measure induced from $m^{*}$-measure on $G^{*}$.

(4) Denoting the Weil topology (on $G$ ) associated with $n$ by $U$, and the topology induced on $G$ from the topology of $G^{*} / H^{*}$ (under the mapping $\phi$ ) by $U_{1}$, we have: (i) $T \leqq U_{1} \leqq U$, (ii) if $W$ is any locally compact topology on $G$ in which $G$ is a topological group and such that $T \leqq W \leqq U$ then $W \leqq U_{1}$.

Proof. The real part of this proof is the proof of Theorem 1.1 above and Andre Weil's theorem about a measure giving rise to a topology. Given the situation described in the hypotheses of this theorem we define $U$ to be the Weil topology associated with $n$ and $G^{*}$ to be the completion of $G$ in the $U$-topology. We then define $H^{*}$ as just above Theorem 2.1. Assertions (1) and (4) above are consequences of Theorem 2.1 while assertions (2) and (3) are merely a restatement of the Weil theorem.

Now considering $G$ in this $U_{1}$-topology, it is a locally compact topological group, and any $U_{1}$-compact neighborhood of the identity generates a locally compact topological subgroup of $G$. We want to show that in general such a subgroup is nontrivial, that is, that it is not a discrete group, and has $m$-measure 0 . For this purpose we need the following theorems. The first of these is a variation of the uniqueness theorem for Haar measure, emphasizing again what Weil has shown [4, pp. 148-149], that the so-called uniqueness theorem is really more of a comparability theorem thaii a uniqueness theorem.

Definition. Let $n$ and $m$ be left invariant measures on a group $G$. We say $n$ 
is a strong refinement of $m$, written $n \gg m$, ff every $n$-measurable set is $m$-measurable and has $m$-measure 0 .

THEOREM 3.2. Let $G$ be a topological group in each of the locally compact topologies, $T$ and $U$, and let the Haar measures of $G$ in these topologies be $m$ and $n$, respectively. If $m \leqq n$ then either $m$ and $n$ are identical (to within a constant factor) or else $m \ll n$.

Proof. Applying Theorem 1.1 we know that $T \leqq U$, and hence that every $U$-compact set is $T$-compact. Hence $\Sigma_{U}$, the $\sigma$-field generated by the $U$-compact sets, is contained in $\Sigma_{T}$, the $\sigma$-field generated by the $T$-compact sets: $\Sigma_{U} \subseteq \Sigma_{T}$.

Let $C$ be any $U$-compact set which is the $U$-closure of a $U$-open set, so that $C$ is both $n$ and $m$-measurable, and $0<n C<\infty, m C<\infty$. Then $m C=k n C$, where $k$ is a real number, $k \geqq 0$. Applying a theorem of Weil [4, p. 149] we know that $m M=k n M$ (the same $k$ as for $C$ ) for all $M \in \Sigma_{U}$, and to prove this theorem it is sufficient to show that either $k=0$ or else every $T$-compact set is $U$-compact.

Now we show that $k>0$ implies every $T$-compact set is $U$-compact. Let $C$ be $T$-compact; being $T$-compact it is $T$-closed, hence it is $U$-closed, and hence it is sufficient to show $C$ is contained in a finite union of $U$-compact sets. Let $D$ be a $T$-compact set containing a $T$-open set; then $C D$ is $T$-compact, hence $m(C D)<\infty$. Now choose $O$ a $U$-open set included in $D$ such that $n O<\infty$. Then (denoting the $n$-inner measure of $E$ by $\left.n_{*} E\right) n_{*}(C O) \leqq n_{*}(C D) \leqq m(C D) / k$. Hence by another theorem of Weil $[4, \mathrm{p} .143] C$ is totally bounded in the $U$-topology; in particular $C$ is included in a finite union of $U$-compact sets. Thus $C$ is $U$-compact, and the theorem is proved.

Our next theorem concerns the existence of nonmeasurable sets. In the case of the real line, or any group $G$ for which $G$ itself is a measurable set, if a set is nonmeasurable then it is so because in some sense it is too irregular. When we omit countability restrictions there may be sets however, for example $G$ itself, that are nonmeasurable because they are "too big." Our condition on a measure that every set must be a union of countably many sets of finite measure is responsible for this. The following theorem says there always exist sets which fail to be measurable by being too irregular ff the topology on $G$ is not discrete, thus describing measure-theoretically the situations in which the topology is discrete.

THEOREM 3.3. Let $G$ be a group, $m$ a measurable left invariant measure on $G$, and $T$ the Weil topology associated with $m$. Then $T$ is discrete ff every subset of an m-measurable set is again m-measurable.

Proof. In case $T$ is discrete there exists an $m$-measurable set $M$ of positive $m$-measure such that $M M^{-1}=(e)$, by definition of the Weil topology on $G$. In this case it is clear that $M=(e)$, so $(e)$ is a set of positive measure. It is then 
trivial to prove the $m$-measurable sets are precisely the finite and countable subsets of $G$ and that the $m$-measure of such a set is the number of points in the set (or that $m$-measure is a constant multiple of this one), so every subset of an $m$-measurable set is again $m$-measurable.

Now suppose that every subset of an $m$-measurable set is again $m$-measurable; we shall prove that $T$ is discrete. We assume the opposite and obtain a contradiction. First we prove that $G$ contains a countable subgroup $H$ such that every neighborhood $O_{1}$ of $e$ in $G$ intersects $H$ in points other than $e$. To see this let $O$ be any totally bounded neighborhood of $e ; O$ contains infinitely many points since $T$ is not discrete and we choose $\left\{x_{n}\right\}$ any countable sequence of points in $O$. We define $H$ to be the subgroup generated (generated in the group sense, that is, without closing up in any topology) by $\left\{x_{n}\right\}$, so obviously $H$ is countable. Every neighborhood $O_{1}$ of $e$ has some translation which contains countably many elements of $\left\{x_{n}\right\}$, since $O$ is totally bounded. If $O_{2}^{-1} O_{2} \subseteq O_{1}\left(O_{2}\right.$ a neighborhood of $e$ in $\left.G\right)$ then some translation $y O_{2}$ contains countably many elements of $\left\{x_{n}\right\}$; choosing $x_{m}, x_{n} \in O_{2}\left(\right.$ and $x_{m} \neq x_{n}$ ) we have $x_{m}^{-1} x_{n} \in\left(y O_{2}\right)^{-1}\left(y O_{2}\right)=O_{2}^{-1} O_{2} \subseteq O_{1}$, and $x_{m}^{-1} x_{n} \in H, x_{m}^{-1} x_{n} \neq e$. Thus every $O_{1}$ intersects $H$ in points other than $e$.

Now we use $H$ to obtain an $m$-measurable $E$ of finite positive measure such that $x E \| y E$ whenever $x$ and $y$ are distinct elements of $H$. We choose one point from each left coset of $H$, letting $E^{\prime}$ be the collection of points chosen. Obviously $\cup_{x} E^{\prime}=G$, where this union is taken over all $x \in H$, and $x E^{\prime} \| y E^{\prime}$ if $x$ and $y$ are distinct elements of $H$. Let $F$ be any measurable set of finite positive measure, and consider the sets $F \cap x E^{\prime}(x \in H)$, each of which is measurable because each is a subset of the measurable set $F$. One of these, which we denote by $E$, must also have positive measure, because there are only countably many of them and their union is $F$.

Now we use $E$ to obtain a contradiction. Weil has shown that $m(x E \ominus y E)$ $\rightarrow 0$ as $x, y \rightarrow e\left({ }^{14}\right)$, which is contradicted by the two properties of $E$ and $H$ : (1) $x E \| y E$ for $x \neq y$, if $x, y \in H$, (2) $H$ intersects every neigh borhood of $e$. Hence the theorem is proved.

The next two theorems are also for the purpose of making sure we can get a nontrivial locally compact subgroup. Their purpose is to consider the relation between $n$-measure and $n_{1}$-measure, and between the $U$-topology and the $U_{1}$-topology, where $n$-measure is the given refinement of the Haar measure $m, U$-topology is the Weil topology associated with $n, U_{1}$-topology is the topology introduced above which is the "closest" locally compact topology to the $U$-topology, and $n_{1}$ is the Haar measure of $G$ in the $U_{1}$-topology. A subgroup can be trivial either by being discrete, or by being too nearly all of $G$.

(14) This statement is contained in Weil's statement [4, pp. 141, 142] that the topology, which is defined by taking the $M M^{-1}$ sets ( $M$ measurable and of positive measure) for neighborhoods of $e$ could equally well be defined by taking the sets $W(\phi, \epsilon)$ (in his notation) for neighborhoods of the identity. 
The next theorem states a condition on $n$ which eliminates the first kind of triviality, and the following theorem gives a condition eliminating the second kind. Although these two theorems do not mention the subgroup we are seeking, the one following them takes care of that.

THEOREM 3.4. Let $G$ be a locally compact topological group with Haar measure $m$ and let $n$ be a measurable left invariant measure on $G$ which is a refinement of $m$. Let $U_{1}$ be the topology described in Theorem 3.1. Then $U_{1}$ is the discrete topology ff every subset of an n-measurable set is again n-measurable.

Proof. First note that this is not a special case of the preceding theorem because the $U_{1}$-topology is not the Weil topology associated with $n$-measure. Now suppose $U_{1}$ is discrete. Then $U$ (the Weil topology associated with $n$ ) is a refinement of $U_{1}$, by Theorem 3.1, and hence is discrete. Hence Theorem 3.3 implies that every subset of an $n$-measurable set is again $n$-measurable.

Next suppose that every subset of an $n$-measurable set is again $n$-measurable. Then, by Theorem 3.3, $U$ is the discrete topology on $G$. Making use of the fact that $G$ is a dense subgroup of $G^{*}$ in the $U^{*}$-topology, and that $U$ is the topology on $G$ induced from the $U^{*}$-topology, it is clear that the $U^{*}$-topology is discrete. Because the natural mapping of $G^{*}$ onto $G^{*} / H^{*}$ is open it follows that the topology on $G^{*} / H^{*}$ is discrete, and hence that the $U_{1}$-topology on $G$ is discrete.

THEOREM 3.5. Let $G$ be a locally compact topological group with Haar measure $m$ and let $n$ be a measurable left invariant measure on $G$ which is a refinement of $m$. Let $n_{1}$ be the Haar measure of $G$ in the $U_{1}$-topology described in Theorem 3.1. Then $n \gg m f f n_{1} \gg m$.

Proof. First suppose $n_{1} \gg m$. Let $O_{1}$ be any $n_{1}$-measurable $U_{1}$-open set; then $O_{1}$ is also $U$-open and $m O_{1}=O$. Let $O$ be any $n$-measurable $U$-open set included in $O_{1}$, so then $m O=O$. Since every $n$-measurable set is contained in a countable union of left translations of such an $O$ it follows that $n \gg m$.

Now suppose $n \gg m$; then by Theorems 1.1 and 3.2 either $n_{1} \gg m$ or $n_{1}=k m$; $k>0$ (and where this equality sign includes the fact that $n_{1}$ and $m$ are defined for exactly the same sets); we shall suppose the latter and obtain a contradiction. Let $G_{0}^{*}$ be any open and closed subgroup of $G^{*}$ which is the union of a countable number of compact sets (such a subgroup can be obtained of course by taking a $U^{*}$-open set whose $U^{*}$-closure is compact, then letting $G_{0}^{*}$ be the subgroup generated by $O^{*}$ ). Now we define two subgroups, $G_{0}$ and $G_{1}$, of $G$ by $G_{0}=G_{0}^{*} \cap G$, and $G_{1}=$ image of $G_{0}$ in $G$ obtained by applying the natural homomorphism of $G^{*}$ onto $G^{*} / H^{*}$ and following that with our natural isomorphism $\phi$ of $G^{*} / H^{*}$ onto $G$. Because of our choice of $G_{0}^{*}$ it is clear that $G_{1}$ is a $U_{1}$-open subgroup of $G, G_{1}$ is $n_{1}$-measurable, $n_{1} G_{1}>0$, and $G_{0} \subseteq G_{1}$.

Let $F$ be a $U_{1}$-closed subset of $G_{1}-G_{0}$ of positive $n_{1}$-measure (which exists, from standard properties of Haar measure) and $F^{*}$ the inverse image of $F$ 
under our homomorphism of $G_{0}^{*} / H_{0}^{*}$ onto $G_{0}$, that is, $F^{*}=F\left(G_{0}^{*} \cap H^{*}\right)$. Then $F^{*}$ is closed in $G_{0}^{*}$ and because of our choice of $G_{0}^{*}, F^{*}$ is $m^{*}$-measurable. Using Theorem 1 of the following paper we see that $F^{*}$ has positive $m^{*}$-measure because

$$
m^{*} F^{*}=\int_{G_{1}} m^{\prime}\left(F_{x}^{*}\right) d n_{1} \geqq \int_{F} m^{\prime}\left(F_{x}^{*}\right) d n_{1}=m^{\prime}\left(G_{0}^{*} \cap H^{*}\right) n_{1} F>0
$$

where $F_{x}^{*}=F^{*} \cap x\left(G_{0}^{*} \cap H^{*}\right)$, and $m^{\prime}$ denotes the Haar measure of $G_{0}^{*} \cap H^{*}$ transplanted to all its cosets in $G_{0}^{*}$ (it is clear that $G_{1}=G_{0}^{*} /\left(G_{0}^{*} \cap H^{*}\right)$ ). Since it is clear that $F^{*} \| G$ we have a contradiction with the fact that $G$ is a thick subgroup of $G^{*}$. Hence this theorem is proved.

THEOREM 3.6. Let $G$ be a locally compact topological group with Haar measure $m$ and let $n$ be a measurable left invariant measure on $G$ which is a refinement of $m$. Let $U_{1}$ be the topology described in Theorem 3.1, let $C_{1}$ be a $U_{1}$-compact set which contains a $U_{1}$-open set, and let $G_{1}$ be the subgroup of $G$ generated by $C_{1}$. Then $G_{1}$, taken in the $U_{1}$-topology, is a locally compact topological subgroup of $G$. $G_{1}$ is not discrete (that is, $U_{1}$-topology is not discrete) ff every subset of an $n$-measurable subset is again n-measuroble and $G_{1}$ has $m$-measure 0 ff $n \gg m$.

Proof. This theorem is merely a compilation of the information given by previous theorems.

\section{BiBLIOGRAPHY}

1. J. L. Doob, Trans. Amer. Math. Soc. vol. 42 (1937) pp. 107-140.

2. K. Kodaira, Proceedings of the Physico-Mathematical Society of Japan (3) vol. 23 (1941) pp. 67-119.

3. S. Saks, Theory of the integral, 2d rev. ed., Warsaw, 1937.

4. A. Weil, L'intégration dans les groupes topologiques et ses applications, Actualites Scientifiques et Industrielles, no. 869, Paris, 1940.

5. J. C. Oxtoby, Trans. Amer. Math. Soc. vol. 60 (1946) pp. 215-237.

UNIVERSITY OF MICHIGAN, ANN ARbor, Mich. 